\title{
Author's Reply: Pulmonary Recruitment Maneuver Reduces Shoulder Pain and Nausea After Laparoscopic Cholecystectomy: A Randomized Controlled Trial
}

\author{
Ebba Kihlstedt Pasquier $^{1}$ (D) $\cdot$ Ellen Andersson ${ }^{1}$
}

Accepted: 22 January 2022/Published online: 20 February 2022

(C) The Author(s) under exclusive licence to Société Internationale de Chirurgie 2022

\section{Response to Letter to the Editor}

We would like to thank the editor for the opportunity to respond to the issues raised in the letter by Dr Ali, Dr Perin and Dr Balasubramanian. We likewise thank Dr Balasubramanian and colleagues for their interest in our paper and for taking the time to express their thoughts.

First, the primary end point of the study was the difference in mean pain intensity score between intervention and control groups regarding overall pain, specifically the evolution of NRS score over time, as expressed in the methods section. Indeed, the statistically significant differences detected between the groups regarded secondary outcomes; shoulder pain and nausea and vomiting. Secondary outcomes are supposedly covered by the power estimation of the primary outcome and ought therefore not be considered exploratory.

Concerning the exclusion of participants after randomization due to ERCP, additional surgical interventions or complications, we did consider including their data in an intention to treat analysis. The main reason such an analysis could not be done was due to logistic reasons. If unforeseen events as those mentioned above occurred, participants generally did not receive the study questionnaire. Due to missing data, an intention-to-treat analysis could not be performed.

\footnotetext{
Ebba Kihlstedt Pasquier

kihlstedt@gmail.com

1 Department of Surgery, Norrköping, Sweden
}

Our rationale for using a higher ASA classification as an exclusion criteria was to prevent inclusion of patients with severe pulmonary or cardiac diseases in the study, to avoid possible adverse effects of the pulmonary recruitment maneuver. Since we previously have performed a similar study on patients undergoing laparoscopic bariatric surgery [1], and especially since many patients undergoing laparoscopic cholecystectomy have a BMI $>30 \mathrm{~kg} / \mathrm{m} 2$, we considered it important to include patients with obesity in this study.

Finally, the WHO analgesic ladder is a pain management strategy proposed for patients with cancer pain and other chronic pain conditions. In our clinical practice, we do seldom use weak opioids in postoperative pain management, but prefer strong opioids, when needed.

We are glad to read that Dr Balasubramanian and colleagues found this study translatable into their daily clinical practice, and we hope they experience positive effects of the pulmonary recruitment maneuver. It is a swift, simple and safe intervention with several possible beneficial outcomes.

\section{Reference}

1. Kihlstedt Pasquier E, Andersson E (2018) Pulmonary recruitment manoeuvre reduces pain after laparoscopic bariatric surgery: a randomized controlled clinical trial. Surg Obes Relat Dis 14(3):386-392. https://doi.org/10.1016/j.soard.2017.11.017

Publisher's Note Springer Nature remains neutral with regard to jurisdictional claims in published maps and institutional affiliations. 